\title{
Dark channels in resonant tunneling transport through artificial atoms
}

\author{
Eduardo Vaz and Jordan Kyriakidis** \\ Department of Physics and Atmospheric Science, \\ Dalhousie University, Halifax, Nova Scotia, Canada, B3H $3 J 5$
}

(Dated: March 25, 2022)

\begin{abstract}
We investigate sequential tunneling through a multilevel quantum dot confining multiple electrons, in the regime where several channels are available for transport within the bias window. By analyzing solutions to the master equations of the reduced density matrix, we give general conditions on when the presence of a second transport channel in the bias window quenches transport through the quantum dot. These conditions are in terms of distinct tunneling anisotropies which may aid in explaining the occurrence of negative differential conductance in quantum dots in the nonlinear regime.
\end{abstract}

PACS numbers: 73.21.La, 73.23.-b, 73.23.Hk

\section{INTRODUCTION}

The properties of electron transport in nanoscopic semiconductor devices are an important diagnostic tool in the basic study of charge and spin degrees of freedom of functional and reliable nanoelectronic devices [1]. In quantum dots defined electrostatically in a two dimensional electron gas [2], both the electron number and the tunnel coupling to the leads are tunable. When high potential barriers separate the quantum dot from the source and drain reservoirs, tunneling to and from the dot is weak and the number of electrons $N$ on the dot is a well-defined integer. In the Coulomb blockade (CB) regime [3], transport proceeds primarily by sequential resonant tunneling events and $N$ fluctuates by one. In the non-equilibrium regime, where more than one transport channel lies within the bias window, a classical transport treatment 4] is no longer appropriate, and a nonequilibrium quantum approach is imperative [5, 6. In this paper, we microscopically derive analytic expressions for the current in the sequential regime through the formalism of the reduced density matrix and generalized master equations for the evolution of the occupation probabilities [7. One of the main motivations is the recent observation of negative differential conductance in theory and experiment [8, 9, 10, 11, 12] in several specific systems. Our goal in the present work is to derive rather general but well-defined conditions for the suppression or even the quenching of current whenever a second channel enters the bias window.

\section{MODEL}

We define our model as a two-dimensional lateral quantum dot [13, 14] weakly coupled to source and drain reservoirs, where the total Hamiltonian is given by

$$
H=H_{S}+H_{Q D}+H_{D}+H_{T}
$$

The source and drain Hamiltonians, $H_{S}$ and $H_{D}$ respectively, are taken to be noninteracting Fermion systems shifted by the bias:

$$
H_{S(D)}=\sum_{s(d)}\left(\epsilon_{s(d)} \pm \frac{1}{2} e V_{B}\right) d_{s(d)}^{\dagger} d_{s(d)}
$$

with $d_{s(d)}^{\dagger}$ and $d_{s(d)}$ a creation and an anihilation operator, respectively, for particles in the source (drain) reservoir.

The quantum dot Hamiltonian in Eq.1a is given by,

$$
H_{Q D}=\sum_{i}\left(\hbar \omega_{i}+e V_{g}\right) c_{i}^{\dagger} c_{i}+V_{\mathrm{int}},
$$

${ }^{*}$ URL: http://soliton.phys.dal.ca 
where the single-particle energies $\hbar \omega_{i}$ are shifted by the applied gate voltage $V_{g}$, and $V_{\text {int }}$ is the interaction among the confined particles, which we leave general in the present work.

Finally, the coupling between the quantum dot and reservoirs is described by the tunneling Hamiltonian,

$$
H_{T}=\sum_{k}\left[\left(\sum_{s} T_{k}^{s} d_{s}+\sum_{d} T_{k}^{d} d_{d}\right) c_{k}^{\dagger}+\text { h.c. }\right],
$$

where h.c. denotes the hermitian conjugate, and where $T_{k}^{s(d)}$ is the tunneling coefficient for a particle tunneling through the barrier between the single-particle states $|s\rangle(|d\rangle)$ in the source (drain) reservoir and $|k\rangle$ in the dot. We assume operators in each subsystem are independent of each other, and that the eigenstates of the system are known. For $V_{\text {int }} \neq 0$ in $(1 \mathrm{c})$, these states are, in general, correlated states - coherent superpositions of Slater determinants.

\section{QUANTUM MASTER EQUATION}

We derive master equations for a non-equilibrium system $\left(V_{S D}>0\right)$ by means of the reduced density matrix, $\rho_{m n}$ of the system [7]. In the Born-Redfield theory [15, 16], the diagonal elements are given by

$$
\dot{\rho}_{m m}=\sum_{n} W_{m n} \rho_{n n}-\sum_{n} W_{n m} \rho_{m m}
$$

The general form of the transition rate $W_{m n}$ from state $|n\rangle$ to $|m\rangle$, where both states are in general many-body correlated states, is given by [17,

$$
W_{m n}=\frac{2 \pi}{\hbar} \sum_{\alpha \beta} \int_{-\infty}^{\infty} d \omega\left[\tilde{A}_{n m}^{\alpha \beta}\left(\omega, \epsilon_{F}-v^{-}\right) B_{\alpha \beta}^{S}\left(\omega+v^{-}\right)+\tilde{A}_{n m}^{\alpha \beta}\left(\omega, \epsilon_{F}-v^{+}\right) B_{\alpha \beta}^{D}\left(\omega+v^{+}\right)\right],
$$

where $v^{ \pm}=e\left(V_{g} \pm V_{B} / 2\right)$, and where

$$
\begin{gathered}
\tilde{A}_{n m}^{\alpha \beta}(\omega, \Omega)=A_{n m}^{\alpha \beta}(\omega) \Theta(\omega-\Omega)+A_{m n}^{\alpha \beta}(\omega) \Theta(\Omega-\omega), \\
A_{n m}^{\alpha \beta}(\omega)=\left\langle n\left|c_{\alpha}^{\dagger}\right| m\right\rangle\left\langle m\left|c_{\beta}\right| n\right\rangle \delta\left(\omega-\omega_{n m}\right)
\end{gathered}
$$

is a generalized quantum dot spectral function with $\omega_{n m}=\omega_{n}-\omega_{m}$ and $\Theta(\omega)$ a step function. The analogous spectral function for the source reservoir at zero temperature is

$$
B_{\alpha \beta}^{S}(\omega)=\sum_{s} T_{\alpha}^{s} T_{\beta}^{s *} \delta\left(\omega-\epsilon_{s}\right)
$$

and $B_{\alpha \beta}^{D}(\omega)=\left.B_{\alpha \beta}^{S}(\omega)\right|_{s \rightarrow d}$.

The first term in the integrand of (3) describes the dot interaction with the source reservoir. This portion of $W_{m n}$ may be written as $W_{m n}^{S+}+W_{m n}^{S-}$ respectively denoting the addition and removal of an electron to or from the dot, corresponding to the two terms of 4a. The second term in (3), describes analogous interactions with the drain reservoir, allowing us to symbolically decompose (3) into four terms,

$$
W_{m n}=W_{m n}^{S+}+W_{m n}^{S-}+W_{m n}^{D+}+W_{m n}^{D-},
$$

denoting the addition and removal of particles from the source and drain reservoirs.

It is useful to define a generalized chemical potential $\mu_{N}^{n}$, as the energy required to add a particle to the $N$-particle ground state, yielding an $(N+1)$-particle system in the $n^{\text {th }}$ excited state $(n=0$ is therefore the usual chemical potential). That is, $\mu_{N}^{n}=E_{N+1}^{n}-E_{N}^{0}$, where $E_{N+1}^{n}$ is the $n^{\text {th }}$ excited state of the $(N+1)$-particle system, and $E_{N}^{0}$ is the ground state of the $N$-particle system. At zero temperature and zero bias, the system is in the $N$-particle ground state when the chemical potential $\mu_{N-1}^{0}$ lies below the Fermi energy of the reservoirs, and $\mu_{N}^{0}$ lies above the Fermi energy.

\section{TRANSITION CONDITIONS}

If we denote by $N$ the number of confined particles at equilibrium, and by $N_{k}$ the number of particles in the state $|k\rangle$, then by an analysis of Eqns. (4a), 4b) and (5), one can deduce that $W_{m n}$ is non-zero only when one or more of 
the following four sets of conditions are satisfied:

$$
\begin{gathered}
N<N_{m}=N_{n}+1,\left(\nu_{N}^{N_{n}}+g^{+}\right)<\left(\Delta_{n m} \pm \frac{1}{2} e V_{B}\right), \\
N \geqslant N_{m}=N_{n}+1,\left(\nu_{N_{n}}^{N-1}+g^{-}\right)>\left(\Delta_{m n} \mp \frac{1}{2} e V_{B}\right), \\
N \leqslant N_{m}=N_{n}-1,\left(\nu_{N_{m}}^{N}+g^{+}\right)>\left(\Delta_{m n} \pm \frac{1}{2} e V_{B}\right), \\
N<N_{m}=N_{n}-1,\left(\nu_{N_{m}}^{N-1}+g^{-}\right)<\left(\Delta_{n m} \mp \frac{1}{2} e V_{B}\right) .
\end{gathered}
$$

Here, $\nu_{N}^{M} \equiv \mu_{M}^{0}-\mu_{N}^{0}$ denotes the difference in chemical potentials between $M$ particles and $N$ particles in the dot. In addition, $g^{+} \equiv \mu_{N}^{0}-\left(\epsilon_{F}-e V_{g}\right)$ denotes the energy difference between the chemical potential of the $N$-particle dot and the chemical potential of the source reservoir, and similarly $g^{-} \equiv\left(\epsilon_{F}-e V_{g}\right)-\mu_{N-1}^{0}$ denotes the energy difference between the chemical potential of the drain reservoir and that of the $(N-1)$-particle dot. The energy difference $\Delta_{m n} \equiv \delta E^{m}-\delta E^{n}$, where $\delta E^{n}$ is the excitation energy of the state $|n\rangle$. That is, if $E_{0}^{N_{k}}$ is the groundstate energy of the $N_{k}$-particle system, then $E^{k}=E_{0}^{N_{k}}+\delta E^{k}$ with $\delta E^{k} \geqslant 0$. The relations $N \lessgtr N_{m}=N_{n} \pm 1$ in Eq. (7) are a consequence of sequential tunneling by (only) a single electron. On the other hand, the relations $\left(\nu_{N_{1}}^{N_{2}}+g^{ \pm}\right) \lessgtr\left(\Delta_{p q} \pm e V_{B} / 2\right)$ are a consequence of resonant tunneling. Moving out of the Coulomb blockade regime relaxes the first set of inequalities while non-Markovian effects relax the second set.

\section{CURRENTS: 1 AND 2 CHANNEL SYSTEMS}

We consider the current through a quantum dot such that electrons tunnel into the dot from the source reservoir, and out of the dot to the drain. The evolution of the diagonal elements of the density matrix (2) for the system depends on both the source and drain reservoirs and can be separated into a contribution due to coupling with the source, and a contribution due to coupling with the drain,

$$
\dot{\rho}_{m m}=\dot{\rho}_{m m}^{S}+\dot{\rho}_{m m}^{D}=\sum_{n}\left[\left(W_{m n}^{S+} \rho_{n n}-W_{n m}^{S+} \rho_{m m}\right)+\left(W_{m n}^{D-} \rho_{n n}-W_{n m}^{D-} \rho_{m m}\right)\right] .
$$

We denote current into the dot through the source barrier by $I^{S}$, and current out of the dot through the drain barrier by $I^{D}$. At long times, the current is proportional to the difference between these two quantities: $I=I^{S}-I^{D}=$ $e\langle\dot{N}\rangle_{S}-e\langle\dot{N}\rangle_{D}$, where, for example, $\langle\dot{N}\rangle_{S}=\sum_{m} N_{m} \dot{\rho}_{m}^{S}$.

Using (8) we obtain,

$$
\begin{aligned}
I^{S} & =e \sum_{m n} N_{m}\left(W_{m n}^{S+} \rho_{n}-W_{n m}^{S+} \rho_{m}\right) \\
I^{D} & =e \sum_{m n} N_{m}\left(W_{n m}^{D-} \rho_{m}-W_{m n}^{D-} \rho_{n}\right),
\end{aligned}
$$

and $I^{S}=-I^{D}$ in the steady-state regime.

We consider the configuration shown in Fig. 1, where the initial equilibrium state of the system is the $N$-particle ground state, and we calculate the steady state currents through the dot for the respective cases of one (Fig. 1 $\mathrm{b}$ ) and two (Fig. 1. c) channels in the transport window. We define three many-body states: $|1\rangle \equiv|N\rangle_{0}$ is the $N$-particle ground state, $|2\rangle \equiv|N+1\rangle_{0}$ is the $(N+1)$-particle ground state, and $|3\rangle \equiv|N\rangle_{1}$ is the $N$-particle first excited state. The transitions between these states are governed in part by Eq. (7).

There will be precisely one channel in the transport window, Fig. 11b, whenever all of the following three inequalities are satisfied:

$$
\begin{gathered}
g^{+}<\frac{1}{2} e V_{B}<g^{-} \\
\frac{1}{2} e V_{B}<\Delta^{N}-g^{+} \\
\frac{1}{2} e V_{B}<\Delta^{N+1}+g^{+},
\end{gathered}
$$

where $\Delta^{N} \equiv E_{1^{\text {stexc }}}^{N}-E_{\text {ground }}^{N}$ is the excitation energy of the first excited state of the $N$-particle system (this excitation can be due to an electron-electron interaction, or due to Zemann spliting in the presence of a magnetic 


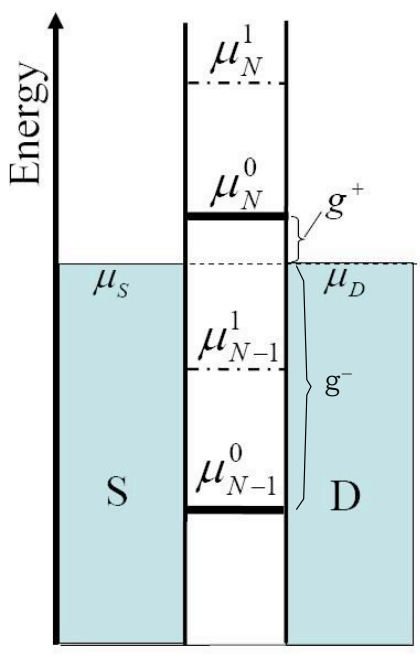

(a)

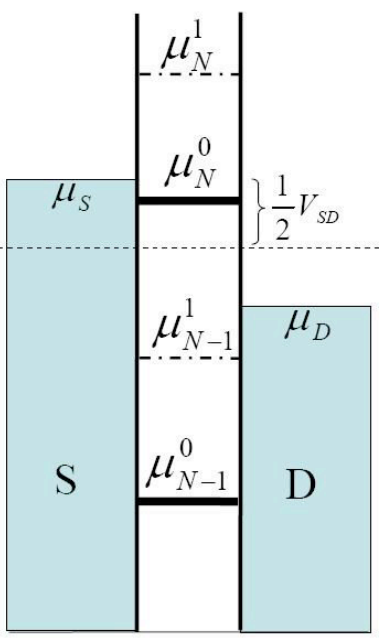

(b)

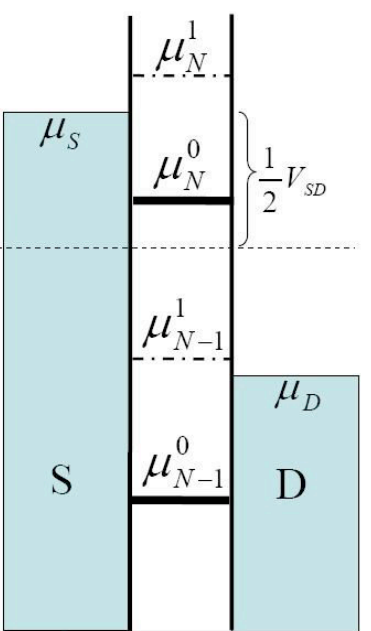

(c)

FIG. 1: Diagram of dot energy regimes. (a) No transport channels in the bias window, (b) single transport channel, $\mu_{N}^{0}$, in the bias window, (c) two transport channels, $\mu_{N}^{0}$ and $\mu_{N-1}^{1}$, in the bias window.

field [18). Assuming initially unit occupation of only the $N$-particle ground state, the infinite coupled set (2) reduces, on application of (7) to two equations:

$$
\begin{aligned}
& \dot{\rho}_{11}=W_{12} \rho_{22}-W_{21} \rho_{11}, \\
& \dot{\rho}_{22}=W_{21} \rho_{11}-W_{12} \rho_{22} .
\end{aligned}
$$

That is, transport involves the ground states of the $N$ and $(N+1)$-particle systems (only), as expected. Solving this coupled set and inserting into (9), the steady state currents are found to be

$$
I_{b}=I_{b}^{S}=I_{b}^{D}=e \frac{W_{21}^{S+} W_{12}^{D-}}{W_{21}^{S+}+W_{12}^{D-}}
$$

which is the standard expression 19, 20, for a two-level steady state dc current.

The two-channel regime, Fig. 1; , is entered upon relaxing condition (10b). If we again assume unit occupation of the $N$-particle ground state as our initial condition, the set (2) now reduces to three equations:

$$
\begin{gathered}
\dot{\rho}_{11}=W_{12} \rho_{22}-W_{21} \rho_{11} \\
\dot{\rho}_{22}=W_{21} \rho_{11}+W_{23} \rho_{33}-\rho_{22}\left(W_{12}+W_{32}\right) \\
\dot{\rho}_{33}=W_{32} \rho_{22}-W_{23} \rho_{33}
\end{gathered}
$$

Transport now involves the ground states of the $N$ and $(N+1)$-particle ground states - states $|1\rangle$ and $|2\rangle$ respectivelyas well as the first excited state with $N$ particles - state $|3\rangle$. (Note that if condition (10c) rather that $10 \mathrm{~b}$ were relaxed, the expressions, and the states involved, would differ.) Solving this coupled set and inserting into (9), the steady state currents are found to be

$$
I_{c}=I_{c}^{S}=I_{c}^{D}=\frac{e W_{21}^{S+} W_{23}^{S+}\left(W_{12}^{D-}+W_{32}^{D-}\right)}{W_{21}^{S+} W_{32}^{D-}+W_{23}^{S+}\left(W_{12}^{D-}+W_{21}^{S+}\right)} .
$$

Upon increasing the bias from zero, the steady-state transport current should change from zero to $I_{b}(12)$, and finally to $I_{c}(14)$. Negative differential conductance will be observed whenever $I_{c}<I_{b}$. That is, an increase in the bias reduces the current. General conditions under which such phenomena occur are explored in the subsequent sections.

\section{ASYMMETRIES}

To explore the relative magnitudes of $I_{b}$ and $I_{c}$, we define two anisotropies. The first is an extrinsic anisotropy characterizing the barrier widths. If we assume tunneling to and from the source is proportional to tunneling to and 
from the drain, we can write

$$
W_{m n}^{S+} / W_{n m}^{D-} \equiv \alpha_{m n}
$$

for all many-body states $|n\rangle$ and $|m\rangle$. Through Eq. (3), these can be related directly to the tunneling coefficients $T_{k}^{d}$, $T_{l}^{s}$ appearing in Eq. (1d). In particular, the noninteracting limit yields

$$
\alpha_{m n} \underset{\text { non-interacting }}{\stackrel{\text { limit }}{\longrightarrow}}\left|T_{k_{0}}^{s_{0}}\right|^{2} /\left|T_{k_{0}}^{d_{0}}\right|^{2},
$$

where $T_{k_{0}}^{s_{0}}$ and $T_{k_{0}}^{d_{0}}$ are the tunneling amplitudes of the resonant states. In general, $0<\alpha<\infty$.

The second anisotropy is intrinsic to the dot and denotes the relative ease with which particles can tunnel to different orbitals. Core orbitals tightly bound to the center of the dot, for example, have weaker coupling to the leads than do orbitals localized around the edge of the dot. for the particular case of the many-body states $|1\rangle,|2\rangle$, and $|3\rangle$ defined above, we write

$$
W_{12}^{D-} / W_{32}^{D-} \equiv \epsilon,
$$

where $|1\rangle$ and $|3\rangle$ are $N$-body states, and $|2\rangle$ is an $(N+1)$-body state. The magnitude of $\epsilon$ will be determined by the spatial extent of the single-particle orbitals involved in the respective transitions. Starting from the $(N+1)$-particle state $|2\rangle, W_{12}^{D-}$ describes an electron tunneling out of the dot, leaving the system in the $N$-particle state $|1\rangle$, whereas $W_{32}^{D-}$ describes a similar process, with the system ending up in state $|3\rangle$. The ration $\epsilon$ depends on the particular orbitals involved.

In the example we give below, $1<\epsilon<\infty$.

\section{QUENCHING OF CURRENT}

Examining the currents $I_{c}$, Eq. 14, and $I_{b}$, Eq. 12, in terms of the anisotropy parameters $\alpha_{m n}$ and $\epsilon$, we find

$$
\frac{I_{c}}{I_{b}}=\frac{(1+\epsilon)\left(1+\alpha_{21}\right)}{\epsilon\left(1+\alpha_{21}+\frac{\alpha_{21}}{\alpha_{23}}\right)} \equiv R \text {. }
$$

For $R<1$, current is suppressed and, in the extreme case of $\alpha_{23} \rightarrow 0$ (i.e., $W_{23}^{S+} \rightarrow 0$ ), the presence of the second channel in the transport window completely quenches the current, $I_{c} \rightarrow 0$. A detail of the Coulomb diamond for which this analysis applies is given in Fig. 2 .

As an illustrative example, we consider a two-dimensional parabolic quantum dot, confining two electrons, with sequential resonant tunneling proceeding by the addition and removal of a third electron. The three states corresponding

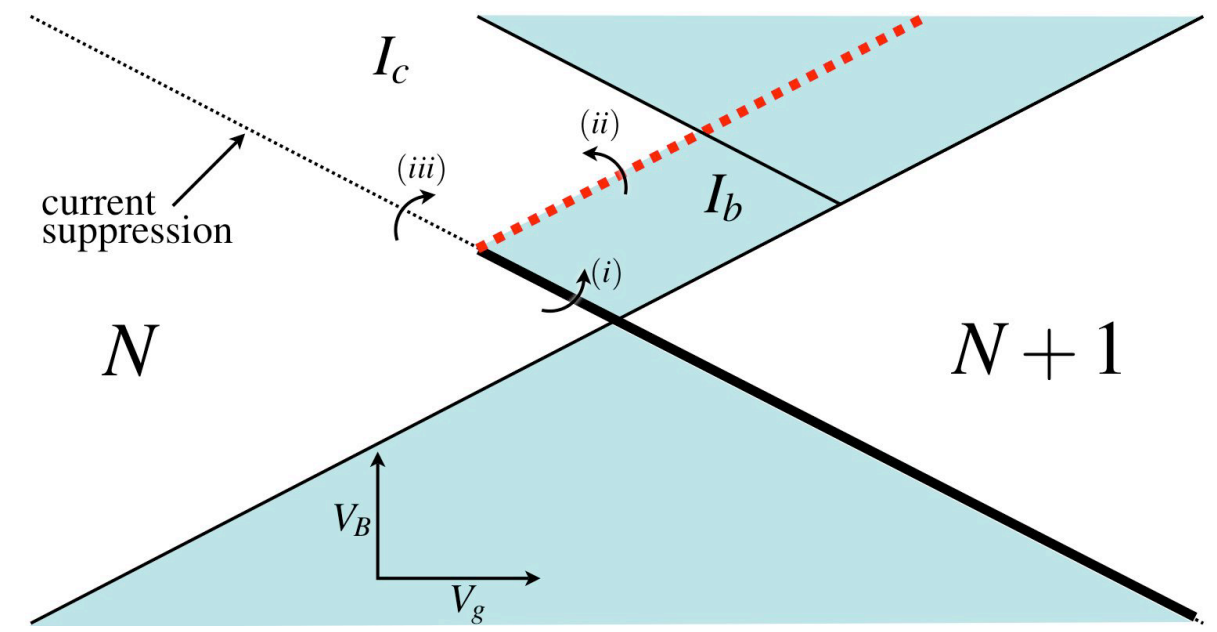

FIG. 2: Coulomb diamond detail showing suppression of ground-state current. the current increases along $(i)$, but may decrease along $(i i)$. In extreme cases, the decrease along $(i i)$ matches the increase along $(i)$, yielding no current along (iii). $I_{b}$ and $I_{c}$ correspond respectively to (b) and (c) of Fig. 1, and their analytic expressions are given in Eqns. (12) and 14 respectively. 


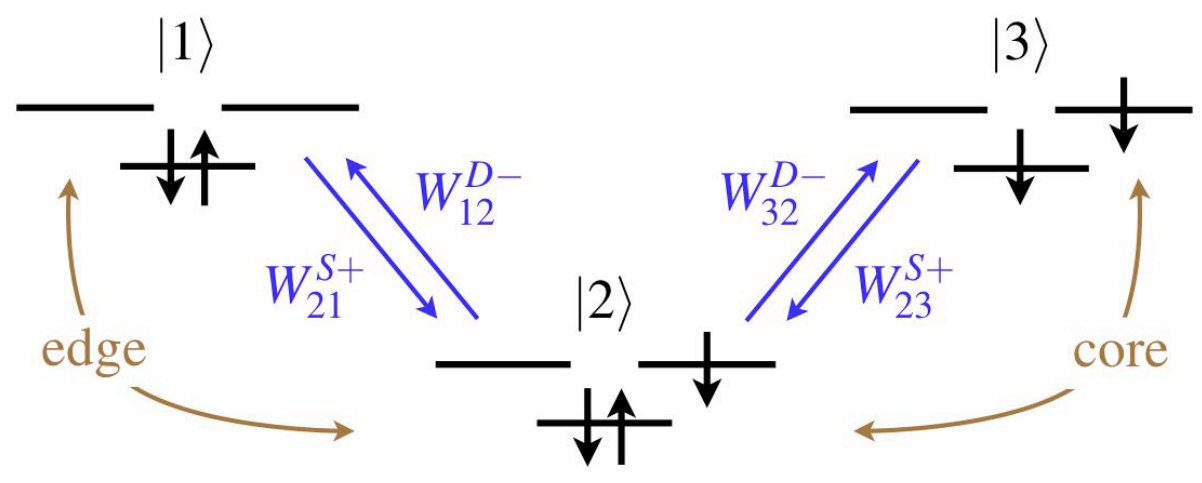

FIG. 3: Configurations capable of quenching ground-state transport. Rates involving states $|1\rangle$ and $|2\rangle$ involve tunneling of an electron into a dot edge-state (more probable) while those involving states $|3\rangle$ and $|2\rangle$ involve tunneling into a dot core-state (less probable).

to Fig. 1F are the ground-state singlet, $|1\rangle$, the lowest-energy triplet, $|3\rangle$, and the three-particle ground state, $|2\rangle$. These respective configurations are shown in Fig. 3. The orbitals can be labeled by two harmonic oscillator indices plus spin, $|m n s\rangle$, where the shell number is given by $m+n=0,1,2, \ldots$, and the angular momentum $L_{z}=n-m=0, \pm 1, \pm 2, \ldots$. With regard to Fig. 3 we have $|1\rangle=|00 \uparrow, 00 \downarrow\rangle,|2\rangle=|00 \uparrow, 00 \downarrow, 01 \downarrow\rangle$, and $|3\rangle=|00 \downarrow, 01 \downarrow\rangle$. The orbital $|01\rangle$ penetrates the barrier [1] to a greater degree than the $|00\rangle$ orbital, and so the tunneling amplitude $T_{00}^{s(d)}$ has smaller magnitude than $T_{01}^{s(d)}$; depending on the geometry and the magnetic field, this difference can be severe. In general, we expect $\epsilon>1$ in Eq. (17).

In the two-channel regime, we look first at the symmetric barrier case, $\alpha_{m n}=1$. Here, suppression of current, $R<1$, Eq. (18), occurs whenever $\epsilon>2$, that is, whenever tunneling into the edge is more than two times as likely as tunneling into the core. In this case, current is never completely quenched; the limiting case $(\epsilon \rightarrow \infty)$ has $R \rightarrow 2 / 3$.

Complete quenching does occur, however, if we additionally have a barrier asymmetry. Specifically, if the source barrier is sufficiently opaque such that tunneling into the core from the source reservoir is suppressed $\left(W_{23}^{S+} \rightarrow 0\right.$, see Fig. 3), but tunneling from the core to the drain is not $\left(W_{32}^{D-} \neq 0\right)$, then $\alpha_{23} \rightarrow 0$ and $I_{c} \rightarrow 0$ even though $I_{b} \neq 0$; the addition of the second transport channel in the bias window suppresses the current completely. Once state $|3\rangle$ becomes occupied, the system is blocked and no other transitions can occur.

This quenching of current is a dynamical blockade: it requires an interplay between both ground and excited states. This is in contrast to effects such as a) spin blockade, where a transition changing the number of electrons by one is accompanied by a change of spin greater than $1 / 2$ [21], or b) blockade due to spin polarized injection and extraction of charge carriers [22, where the current is quenched due to the Pauli exclusion principle.

The quenching of current will be destroyed whenever the spin-flip plus orbital decay rate is large compared to the dwell time of the tunneling electron. In this case, a direct transition from $|3\rangle$ to $|1\rangle$ may occur by emission of a phonon and simultaneous absorption of spin through nuclear baths, spin-orbit coupling, or other spin-symmetrybreaking process. Likewise, strong Coulomb correlations will generally open up additional tunneling pathways, likely involving edge orbitals, and this too will destroy the quenching of current.

\section{CONCLUSIONS}

In conclusion, we have derived a set of general yet well-defined conditions for quenching of transport through a multichannel quantum dot. We find that under certain tunneling anisotropies the presence of a second transport channel may decrease the current through the dot. Specifically, if the tunneling rates to or from core states are less than half than the rates to or from edge states, the current may be suppressed. Complete quenching of the current may occur if, in addition to the edge/core anisotropy, an anisotropy between source and drain barriers is also present. The presence of these dark channels is a measure of the lifetime of excited states.

Finally, although the present work focused on the two-electron case, the general phenomena should appear in numerous locations throughout the Coulomb diamonds; it can generally appear whenever ground-state tunneling transitions involve edge orbitals, and excited-states tunneling involves core orbitals, and asymmetric source and drain barriers are present. 


\section{Acknowledgments}

This work was supported by NSERC of Canada and the Canadian Foundation for Innovation. JK additionally acknowledges discussions with the Institute for Microstructural Sciences of NRC Canada.

[1] L. P. Kouwenhoven, D. G. Austing, and S. Tarucha, Rep. Prog. Phys. 64, 701 (2001).

[2] R. C. Ashoori, Nature 379, 413 (1996).

[3] C. W. J. Beenakker, Phys. Rev. B 44, 1646 (1991).

[4] C. Jacobini and P. Lugli, The Monte Carlo Method for Semiconductor Device Simulations (Springer-Verlag, New York, 1989).

[5] J. Shah, Hot Carriers in Semiconductor Nanostructures: Physics and Applications (Academic Press inc., Boston, 1992).

[6] E. Schöll, Theory of Transport Properties of Semiconductor Nanostructures (Chapman and Hall, London, 1998).

[7] K. Blum, Density Matrix Theory and Applications (Springer, New York, 1996).

[8] M. C. Rogge, B. Harke, C. Fricke, F. Hohls, M. Reinwald, W. Wegscheider, and R. J. Haug, Phys. Rev. B 72, 233402 (2005).

[9] M. C. Rogge, F. Cavaliere, M. Sassetti, R. J. Haug, and B. Kramer, New J. Phys. 8, 298 (2006).

[10] F. Cavaliere, A. Braggio, J. T. Stockburger, M. Sassetti, and B. Kramer, Phys. Rev. Lett. 93, 036803 (2004).

[11] M. H. Hettler, H. Schoeller, and W. Wenzel, Europhys. Lett. 57, 571 (2002).

[12] A. Thielmann, M. H. Hettler, J. Konig, and G. Schon, Phys. Rev. B 71, 045341 (2005).

[13] L. Jacak, P. Hawrylak, and A. Wójs, Quantum dots (Springer-Verlag, Berlin, 1998).

[14] J. Weis, R. J. Haug, K. v. Klitzing, and K. Ploog, Phys. Rev. Lett. 71, 4019 (1993).

[15] A.G. Redfield, IBM J. Res. Dev. 1, 19 (1957).

[16] M. Toutounji, J. Chem. Phys. 123, 244102 (2005).

[17] E. V. Vaz, Master's thesis, Dalhousie University (2006).

[18] R. Hanson, L. M. K. Willems Van Beveren, J. M. Elzerman, I. T. Vink, and L. P. Kouwenhoven, Phys. Rev. B 70, 241304 (2004).

[19] Y. Meir and N. S. Wingreen, Phys. Rev. Lett. 68, 2512 (1992).

[20] A. Thielmann, M. H. Hettler, J. Konig, and G. Schon, Phys. Rev. B 68, 115105 (2003).

[21] A. K. Hüttel, H. Qin, A. W. Holleitner, R. H. Blick, K. Neumaier, D. Weinmann, K. Eberl, and P. Kotthaus, Europhys. Lett. 62, 712-718 (2003).

[22] J. Kyriakidis, M. Pioro-Ladriere, M. Ciorga, A. S. Sachrajda, and P. Hawrylak, Phys. Rev. B 66, 035320 (2002). 